\title{
Patterned Growth in Extreme Environments
}

\author{
Penelope Boston \\ Department of Earth \\ and Environmental Science \\ New Mexico Tech \\ Socorro, NM 87801 \\ E-mail: pboston@nmt.edu
}

Keith Schubert

Department of Computer Science and Engineering

CSU San Bernardino

San Bernardinio, California 92407-2393

Email: schubert@csci.csusb.edu

\author{
Jane Curnutt \\ Department of Computer Science \\ and Engineering \\ CSU San Bernardino \\ San Bernardinio, California 92407-2393 \\ Email: jcurnutt@r2labs.org
}

\author{
Ernesto Gomez \\ Department of Computer Science \\ and Engineering \\ CSU San Bernardino \\ San Bernardinio, California 92407-2393 \\ Email: egomez@csci.csusb.edu
}

\begin{abstract}
In the search for life on Mars and other extraterrestrial bodies, one of the biggest problems facing us is, how do we recognize life or the remains of ancient life when we find it? We will need to recognize residual patterns left by life. One approach to recognizing these kinds of patterns is look at patterns created and left by life in extreme environments here on Earth.
\end{abstract}

\section{INTRODUCTION}

A key aspect of planning a space mission, is to set scientific mission objectives, with the ability to adapt them based on observations and mission situations. The search for extraterrestrial life is a major scientific objective, but the exact nature of that life and how to confirm it is a major debate. A further problem in the search for life in space is how to do we select the areas that we want to investigate more intensively? Photo surveys, geology, and knowledge of biology here on Earth can get to a likely area, but, unlike the Martian dust devils [NASA(2005)], there is no motion to point you in the right direction from there.

We use life in extreme environments on Earth as analogs for the kinds of life that we could encounter in space. We propose using these analogs to create a series of templates which could be used to indicate areas that might be worth deeper investigation. In resource limited environments, organisms grow in patterns that are self-enforcing and exhibit hysteresis [Catena(1999)], [Meron et al.(2004)], [Hardenberg et al.(2001)] which can be used to recognize them and their fossils at a distance. Particularly on Mars, as the environment became less hospitable, extremophiles similar to Earth's were likely the last to survive, and should be the easiest to find. Among the techniques that have been used to model these patterns are evolutionarily stable strategies in game theory and differential equations [Klausmeier(1999)], [HilleRisLambers et al.(2001)], [Thiéry et al.(1995)], [Hardenberg et al.(2001)].

While good results have been generated using differential equations, they require tuning of the parame- ters and experience in mathematical and numerical techniques to obtain valid results. In this work we developed cellular automata that produce similar predictions to the differential equation models, while preserving the rapid modeling and hypothesis testing of cellular automata. Similar models can also be applicable to group animal behavior [Couzin et al.(2002)], [Hoare et al.(2004)], [Krause \& Tegeder(1994)], [Pitcher et al.(1996)]. Our method for deriving rules for cellular automata from observed data in organism growth patterns accounts for soil nutrients, water, root growth patterns, and geology allowing scientists to easily examine the effects of modifying conditions without damaging the environment. We apply this model to identify factors affecting patterning with respect to growth, die-out, and stabilization in extreme environments. We compare the results of our model with biovermiculation microbial mats growth in acid caves, and cyanobacteria growth in Zzyzx, CA. These models could be used to rapidly check data from space missions to rate the potential of various locations of containing life or fossils.

\section{BACKGROUND}

In the 1940s, John von Neumann developed the first cellular automata, while working on the self-replicating systems biological problem [Wolfram(2002)]. In 1970, John Conway developed his Game of Life, a two dimensional cellular automaton that exhibited aspects of both order and randomness [Gardner(1970)]. In 1983, Stephen Wolfram published the first of many papers on cellular automata. His research into this area of mathematics culminated in 2002 with the publication of his book, A New Kind of Science [Wolfram(1985)], [Wolfram(2002)].

\section{Cellular Automata}

A cellular automaton (CA) is a computational model that is discrete in both space and time. Essentially we divide 
space into boxes called cells, and only calculate their values at discrete time using a set of fixed rules. A state could represent anything, and in our case it represents the amount of water, nutrients and the biomass. The rules describe how the organism grows or dies in the presence of the water, nutrients and competition from other organisms. CA rules are not usually expressed as formulas, rather they are visual, such as drawing pictures of the neighboring cells and then labeling the next state. Cellular automata have been used to study growth and patterns in forests, arid desert environments, preditor-prey problems, and sea shells. It has also been used to study areas as diverse as epidemiology and linguistics [Couzin et al.(2002)], [Hoare et al.(2004)], [Krause \& Tegeder(1994)], [Pitcher et al.(1996)].

A state could represent anything, and in our case it represents the amount of water, nutrients and the biomass. The rules describe how the organism grows or dies in the presence of the water, nutrients and competition from other organisms. CA rules are not usually expressed as formulas, rather they are visual, such as drawing pictures of the neighboring cells and then labeling the next state. To avoid tedium, we group similar cell patterns using heuristics discerned from discussions with biologists and born out in practice.

Typically CA are deterministic, do not use the current value of the state in the calculation of the next state, and use only one state variable. We consider a generalization of this in which these constraints are relaxed. For this paper, we only relax the determinism constraint, as this allows for more realistic looking results.

Consider the following simple transition rule.

If 3 or 4 of the cells in a neighborhood $( \pm 1$ row and/or column) of a cell are 1 then the next state for the cell is 1 .

This is illustrated by the transition of cell $\mathrm{S}$ :

Time $t_{0}$
\begin{tabular}{|c|c|c|}
\hline 0 & 1 & 1 \\
\hline 1 & $\mathrm{~S}$ & 0 \\
\hline 0 & 0 & 0 \\
\hline
\end{tabular} $\Rightarrow$\begin{tabular}{|l|l|l|}
\hline 0 & 1 & 1 \\
\hline 1 & 1 & 0 \\
\hline 0 & 0 & 0 \\
\hline
\end{tabular}

Thus we only need to specify the necessary value for biomass to enable organism growth, which value of the sum allows organism survival, and which value will result in organism death.

Such rules are deceptively simple, but can lead to very complicated results. We allowed the neighborhood to vary to simulate plant root systems and other nutrient effects. We also allowed the value of the sum to be specified as a list of numbers or a single interval.

To show how this can relate to a case of crowding, consider the following simple rules. An organism is in a hostile environment. It can live if it has no more than 6 neighbors. Above that number, resource competition is too high. A new organism will grow if 2 or 3 of the neighboring squares have organisms (it takes at least 2 to generate a new organism, but the new one needs room- i.e. few neighbors). Start with a row of five organisms and an interesting thing happens, as you can
Fig. 1. Top graphic shows the first ten time periods for the rules: if neighbors $=2$ or 3 then grow, if neighbors $>6$ then die, else stay same. Bottom graphic shows the final pattern for these rules.
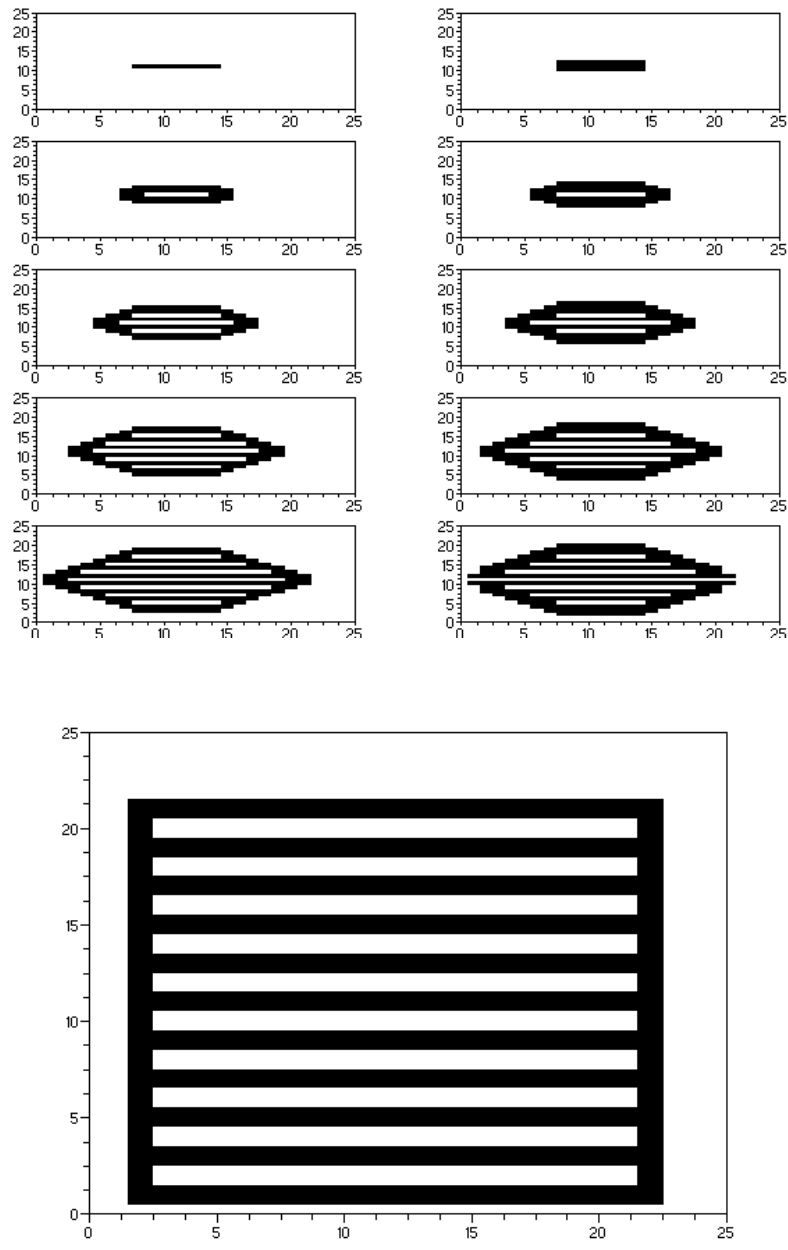

see in Figure 1. The ten small figures show the first 10 time periods, the last one is the area after 40 time periods, when it is stable. If you allow life to be supported then the lines continue to fill the region. None can grow in the "dead zones" once they are established, and placing a living organism there will cause them to die (it will have too many neighbors). This self-enforcing nature of the pattern is the basis of the maze-like growth of plants in extreme environments. More complicated rules that fit more realistic situations generate patterns that imitate the striking structures found in nature.

\section{Cellular Automata and Partial Differential EQUATIONS}

Various methods have been researched for directly transforming spatial partial differential equations into cellular automata models through approximation techniques [Strader(2008)]. In Section IV-A, two methods of transforming a general partial differential equation into a cellular automata rule are shown. In Section IV-B the methods are then analyzed using the Z-transform to find the theoretical constraints of 
stability. Section IV-C illustrates convergence maps of the cellular automata models created from multiple simulation runs. This information results in a set of guidelines that can be used to create faster simulations that end in convergence.

\section{A. Transformation Using Euler's Methods}

Two methods were created of directly transforming differential equations into cellular automata. First a generalized partial differential equation was derived from surveying biological partial differential equations such as desert vegetation patterns (Eqs. 1 and 2) [Meron et al.(2004)] [Hardenberg et al.(2001)],

$$
\begin{aligned}
\frac{\partial n}{\partial t}= & \frac{y w}{1+\sigma w} n-n^{2}-\mu n+\nabla^{2} n \\
\frac{\partial w}{\partial t}= & p-(1-\rho n) w-w^{2} n+\delta \nabla^{2}(w-\beta n)- \\
& v \frac{\partial(w-\alpha n)}{\partial x}
\end{aligned}
$$

Fick's law on population density [Shi(2008)], and predator prey models [Savill \& Hogeweg(1999)]. The following is the general differential equation:

$$
f\left(u_{i, j}\right)=\frac{\partial u}{\partial t}=m\left(u_{i, j}\right)+\nabla_{x}^{2} n\left(u_{i, j}\right)+\nabla_{x} o\left(u_{i, j}\right)
$$

In this equation, $u$ will be the values that are simulated. The subscript $i$ is the time index of $u$ and $j$ is the space index. Most of the surveyed differential equations could be broken up into three groupings of terms based upon whether the Laplacian or gradient operator was applied to them. The terms can be substituted into the $m\left(u_{i, j}\right), n\left(u_{i, j}\right)$, and $o\left(u_{i, j}\right)$ functions.

Once a differential equation is described in this general form, it can easily be transformed to a cellular automata by two different methods. The three point formula is first applied to the general form eliminating the $\nabla_{x}^{2}$ and $\nabla_{x}$ operators. The Forward Euler's method, which is the simpler of the two methods used, can then be applied producing the following formula:

$$
\begin{aligned}
u_{i+1, j}= & u_{i, j}+h_{t}\left(m\left(u_{i, j}\right)+\right. \\
& \frac{n\left(u_{i, j+1}\right)-2 n\left(u_{i, j}\right)+n\left(u_{i, j-1}\right)}{h_{x}^{2}}+ \\
& \left.\frac{o\left(u_{i, j+1}\right)-o\left(u_{i, j-1}\right)}{2 h_{x}}\right)
\end{aligned}
$$

The Backward Euler's method, which is more stable than the Forward Euler's method, can be applied instead giving the formula:

$$
\begin{aligned}
u_{i+1, j}= & u_{i, j}+\frac{h_{t}}{1-\left.h_{t} \frac{\partial m(u)}{\partial u}\right|_{i, j}}\left(m\left(u_{i, j}\right)+\right. \\
& \frac{n\left(u_{i, j+1}\right)-2 n\left(u_{i, j}\right)+n\left(u_{i, j-1}\right)}{h_{x}^{2}}+ \\
& \left.\frac{o\left(u_{i, j+1}\right)-o\left(u_{i, j-1}\right)}{2 h_{x}}\right)
\end{aligned}
$$

Equations 4 and 5 are in fact cellular automata rules because they state that the value of $u$ at space $j$ for the next time period is equal to $u$ 's current value plus or minus some factors of its neighboring values $u_{i, j+1}$ and $u_{i, j-1}$. It is now simple to transform a general differential equation into a cellular automata rule by substituting $m\left(u_{i, j}\right), n\left(u_{i, j}\right)$, and $o\left(u_{i, j}\right)$ in the general differential equation form into either the Forward or Backward formulas. It should also be noted that these equations contain two new important variables as the result of the approximations. The variable $h_{x}$ is the step size for space and $h_{t}$ is the step size for time.

\section{B. Theoretical Stability Constraints}

Equations 4 and 5 were analyzed using the Z-transform to create constraints on stability. The analysis was done only on a general form that used linear terms. The following equation was used to represent the general linear form :

$$
f(u)=a_{1} u+b_{1}+\nabla_{x}^{2}\left(a_{2} u\right)+\nabla_{x}\left(a_{3} u\right)
$$

One can assign parts of the equations to the following functions of Equation 3: $m(u)=a_{1} u+b_{1}, n(u)=a_{2} u$, and $o(u)=a_{3} u$. Here the $a$ and $b$ terms are simply coefficients. After the Z-transform was used on Forward and Backward Euler transformed versions of Equation 6, the equation was solved for $U_{j}$. The $i$ index is eliminated in the Z-transform. According to Z-transform theory, if the pole and zero values of $z$ are within the unit circle, then the equation should remain stable. Therefore, constraints of stability can be found by setting the poles and zeros values to less than one. The zero constraint created was the same for both the Forward and Backward Euler's formulas:

$$
\begin{aligned}
1> & \mid \frac{-1}{2 b_{1} h_{x}^{2}}\left(\left(2 a_{2}-a_{3} h_{x}\right)\left(U_{j-1}\right)+\right. \\
& \left.\left(2 a_{2}+a_{3} h_{x}\right)\left(U_{j+1}\right)\right) \mid
\end{aligned}
$$

The poles constraint for the Forward Euler's was:

$$
1>\left|1+a_{1} h_{t}-\frac{2 a_{2} h_{t}}{h_{x}^{2}}\right|
$$

The poles constraint for the Backward Euler's was slightly different due to the dividing factor that is part of the Backward Euler's equation:

$$
1>\left|1+\frac{a_{1} h_{t}}{1-a_{1} h_{t}}-\frac{2 a_{2} h_{t}}{\left(1-a_{1} h_{t}\right) h_{x}^{2}}\right|
$$

The pole constraints demonstrate that there needs to be a balancing between $h_{t}$ and $h_{x}$. If one is too big relative to the other term, the right hand side will become larger than one, making the formula become unstable.

\section{Convergence Maps and Optimum Convergence}

Simulations were run for the Forward and Backward Euler's formulas using Scilab and a one dimensional cellular automata. The results were combined and graphed onto convergence maps like Figure 2. In this figure simulations were plotted based upon the $h_{x}$ and $h_{t}$ values used for the Backward Euler's equation. A blue dot represents a simulation that ends in convergence, a green dot represents a simulation that ended in divergence, and a red dot is a simulation that hit the maximum 
number of iterations alloted without ending in convergence or divergence.

Both the Forward and Backward Euler's maps had a small stripe of convergence in the lower left area. The Backward Euler's map also had an additional area of convergence in the top right, but this area appeared to be an artifact of the Backward Euler's dividing factor. The striped area of convergence was of interest because as the space step size changed, the final convergence values changed. The convergence areas did not change for different time step sizes.

Fig. 2. Convergence map composed of 10,000 simulations with varying $h_{t}$ and $h_{x}$ values for the Backward Euler's function. The black lines represent the pole constraints.

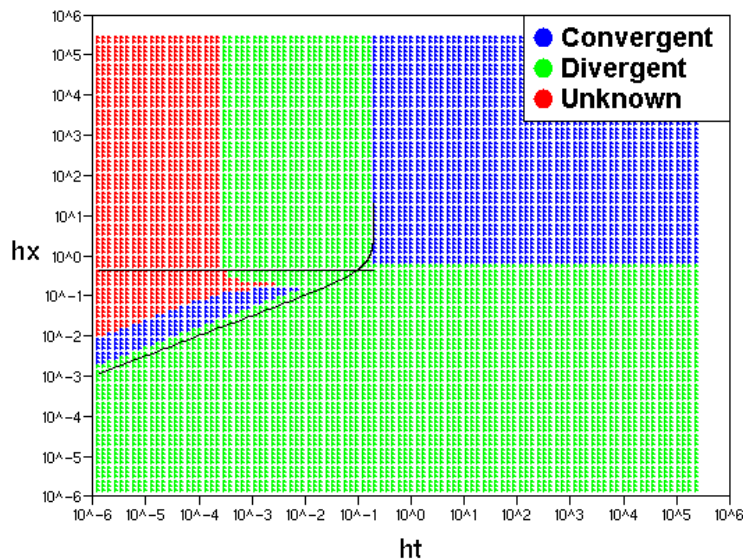

Figure 2 also shows the pole constraints graphed in black. Through multiple simulations it was found that the area of convergence closely matched the area created by the poles constraints, but not exactly. The difference between the actual area of convergence and the pole constraints appeared to be a constant. Tests also showed that the bottom of the area of convergence converged about ten times faster than the top.

Figure 3 contains modified zero constraints in magenta. These constraints required substitutions be made for the $U_{j+1}$ and $U_{j-1}$ terms, which are variables that could not be graphed in the convergence maps. Simulations showed that most of the time that the intersection of the lower pole constraint with the upper zero constraint could be used as an approximation for the maximum $h_{t}$ value for convergence. This information led to a set of guidelines that used the intersection for finding the maximum $h_{t}$ value. A lesser $h_{t}$ value could then be used in the lower pole constraint formula to find an $h_{x}$ value. This would produce an $h_{x}$ and $h_{t}$ pair that would converge quickly because it was a simulation at the bottom of the area of convergence. Hopefully the direct transformation methods and convergence guidelines can eventually be used in a software tool that will help preform pattern simulations for biologists.

\section{EXTREMOPHILES}

Extremophiles form patterns based on their own biology and the environmental conditions they exist in. The CA
Fig. 3. Convergence map for the Backward Euler's function showing the pole constraints in black and the modified zeros constraints in magenta.

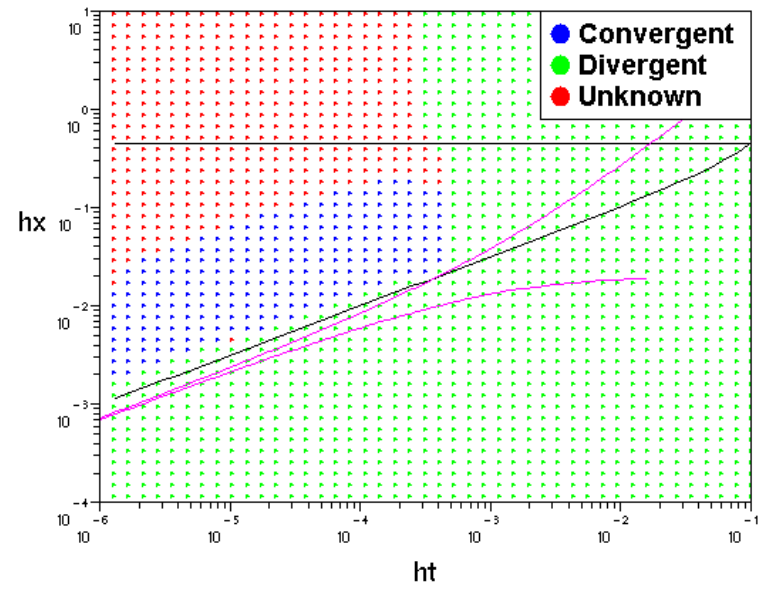

models are adapted to the biological and geological conditions found on Earth that are most likely to match those on Mars. Cyanobacteria and biovermiculations have been identified as part of our work on NASA's Spaceward Bound as likely to be similar to life on Mars.

\section{A. Cyanobacteria}

Cyanobacteria are aquatic, photosynthetic bacteria and are notable for many reasons, including being the oldest fossils, the original producers of atmospheric oxygen, the source of much of our oil, and an ability to grow in extreme environments (including Antarctica). The cyanobacteria in Zzyzx are fossils, but preserve the structured growth we are considering. Given their pivotal and early role on the Earth, similar organisms are conceivable on Mars, and their easy identification is important for biological objectives on Mars.

Figure 4 shows a typical cyanobacteria fossil. These patterns were fairly dense, so we used a neighborhood with a range of 1 square (1 square in all directions for a total of 8 neighbors). Cyanobacteria often grow in blooms, so we modeled this using growth on having 3 neighbors only. We also allowed random death with probability of $10 \%$. The resulting simulation is shown on the right in Figure 4, and shows much of the same structure as is seen in the actual fossil on left in Figure 4.

\section{B. Biovermiculations}

Biovermiculations are microbial mats composed of bacteria, extracellular polysaccharide slime, embedded clay and other particles, in situ precipitated minerals (e.g. sulfur and gypsum), and even some small invertebrates like mites and nematodes. Biovermiculations exist in a wide variety of chemical and physical subsurface settings including sulfuric acid caves, mines, carbonate caves, lavatubes, and even Mayan ruins.

Investigators have identified them from sulfuric acid caves, see [Hose et al.(2000)]. More recently, observers have begun 
Fig. 4. Top Picture is of cyanobacteria fossils from Zzyzx, CA. Bottom graphics are simulated cyanobacteria at $t=5$ (left) and $t=40$ (right).

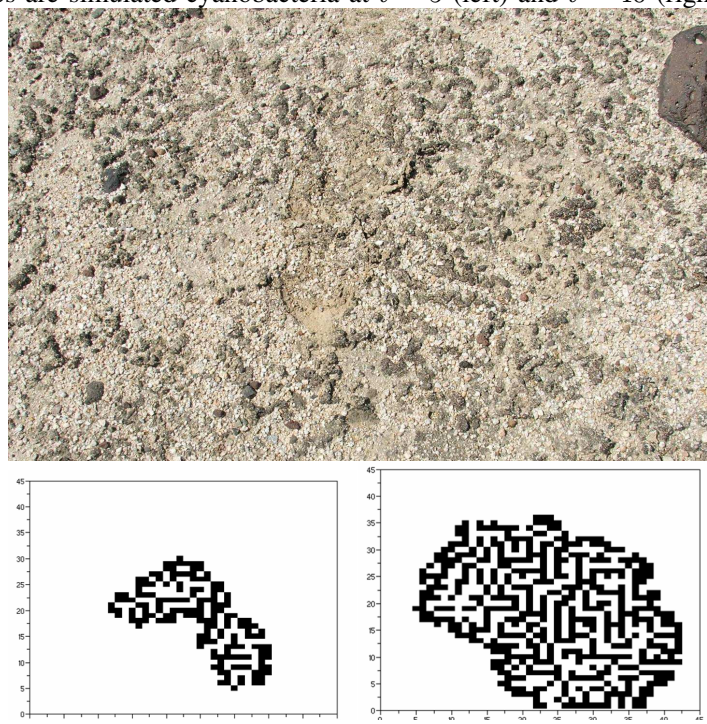

to see them in a wide variety of chemical and physical subsurface settings including mines, carbonate caves, lavatubes, and even Mayan ruins.

These structures are interesting because of their intrinsically intriguing biology and geochemistry, the distinctive growth patterns that they exhibit, and also because they may be a highly distinctive biosignature that could be interpretable in extraterrestrial settings based on gross morphology [Boston et al.(2001)]. Interest in the biovermiculations has grown as better methods of studying such structures has become available. They also provide a model system of a biomat that might occur on the interiors of various cave types on Mars. Lavatubes have been identified on Mars [Boston(2004)], and more recently confirmed in a more elaborate study [Cushing et al.(2007)]. Mechanisms to create solutional caves in evaporite mineral deposits on Mars have also been proposed [Boston et al.(2006)]. Such potential subsurface habitats could conceivably house or have housed microbial populations on Mars and left traces similar to those found in geomicrobiological communities in Earth's subsurface.

The patterning of biovermiculation growth is still a mystery. To understand it will require simulations that test different sets of rules enabling us to arrive at a good pattern match for the microbial mat growth that we are observing in nature. Such simulations will then be correlated with actual pattern examples from cave walls and other occurrences. Figure 5 shows an area that has biovermiculations so thick that they become the solid mat, but there are weird uninhabited areas that follow the rock curvatures. These are not simple water pathways that have prevented growth, so their origin is unknown. In figure 5, no nutrient or water differentiation was induced, only crowding rules, and an ability to pull nutrients from surrounding cells. The result was the depleted region in the middle. Note the shape has many indents, like the actual system in Figure 5.
Fig. 5. Left picture is biovermiculation with discontinuities. Bottom graphic is simulated biovermiculation growth.
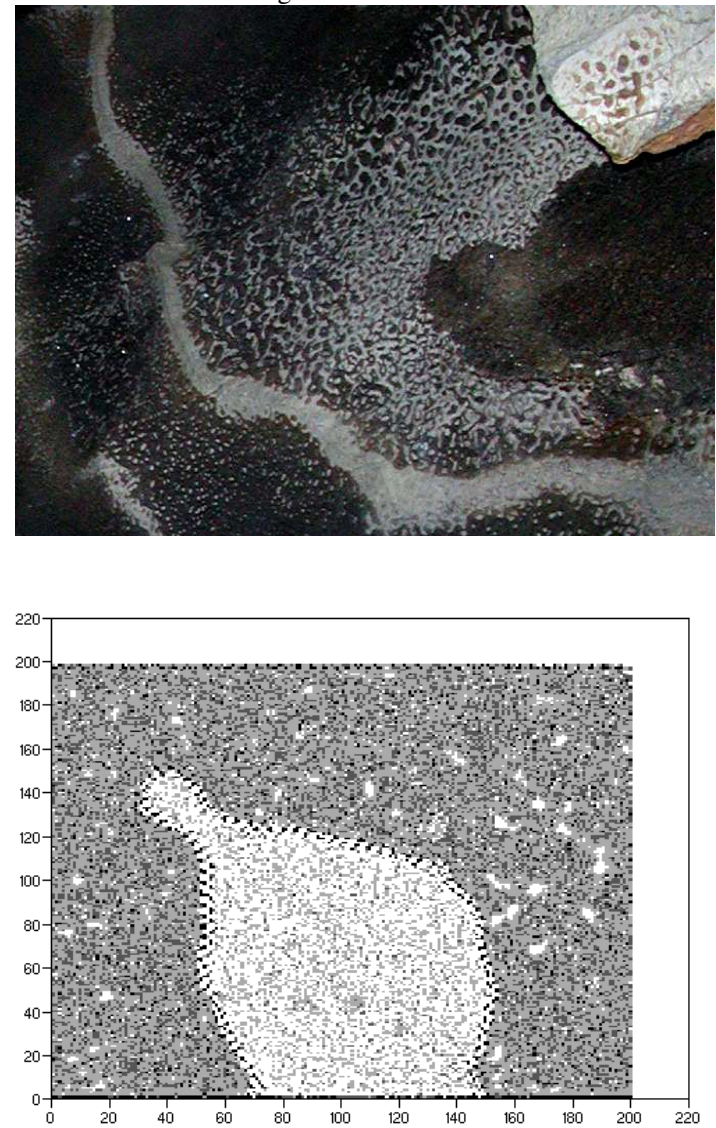

\section{CONCLUSIONS AND FUtURE WORK}

The patterns of microbial extremophile growth, developed using cellular automata, can provide starting templates for the types of patterns we may see in lavatubes and caves on Mars. These patterns could provide indicators of life similar to the patterns that indicated water flow and possible "ponds" on the surface of Mars. These templates and the ability to rapidly identify high probability areas for detecting life will be vital to planning future Mars missions.

\section{REFERENCES}

[NASA(2005)] Press Release Images: Spirit, http://marsrovers.nasa.gov/gallery/press/spirit/20050819a.html, 19August-2005

[Catena(1999)] Catena vol. 37, 1999. Special issue on banded vegetation

[Gardner(1970)] M. Gardner, "The Fantastic Combinations of John Conway's New Solitaire Game 'Life',' Scientific American, vol. 223, pp. 120-123, 1970 .

[Boston(2004)] P.J. Boston. Encyclopedia of Cave and Karst Science, chapter Extraterrestrial Caves, pages 355-358. Fitzroy-Dearborn Publishers, Ltd., London, UK, 2004.

[Boston et al.(2006)] P.J. Boston, L.D. Hose, D.E. Northup, and M.N. Spilde. The microbial communities of sulfur caves: A newly appreciated geologically driven system on Earth and potential model for Mars. In R. Harmon, editor, Karst Geomorphology, Hydrology, \& Geochemistry, pages 331344. Geological Soc. Amer., 2006. 
[Boston et al.(2001)] P.J. Boston, M.N. Spilde, D.E. Northup, L.A. Melim, D.S. Soroka, L.G. Kleina, K.H. Lavoie, L.D. Hose, L.M. Mallory, C.N. Dahm, L.J. Crossey, and R.T. Schelble. Cave biosignature suites: Microbes, minerals and mars. Astrobiology Journal, 1(1):25-55, 2001.

[Klausmeier(1999)] Klausmeier CA. Regular and irregular patterns in semiarid vegetation. Science, (284):1826-1828, 1999.

[Savill \& Hogeweg(1999)] N. J. Savill and P. Hogeweg, "Competition and Dispersal in Predator-Prey Waves," Theoretical Population Biology, vol. 56, pp. 243-263, 1999.

[Shi(2008)] J. Shi, (2008) Partial Differential Equations and Mathematical Biology. [Online]. Available: http://www.resnet.wm.edu/ jxshix/math490/lecture-chap1.pdf

[Strader(2008)] B. Strader, "Simulating Spatial Partial Differential Equations with Cellular Automata," M. CS. thesis, CSU San Bernardino, San Bernardino, CA, USA, Dec. 2008

[Wolfram(1985)] S. Wolfram. "Twenty Problems in the Theory of Cellular Automata." Physica Scripta, iss. T9, pp. 170-183, 1985.

[Wolfram(2002)] S. Wolfram, A New Kind of Science, Wolfram Media Inc., 2002.

[Couzin et al.(2002)] I.D. Couzin, J. Krause, R. James, G.D. Ruxton, and N.R. Franks. Collective memory and spatial sorting in animal groups. $J$. Theor. Biol., (218):1-11, 2002.

[Cushing et al.(2007)] G.E. Cushing, T.N. Titus, J.J. Wynne, and P.R. Christensen. Themis observes possible cave skylights on mars. Geophysical Research Letters, 34(L17201), 2007.

[Dunkerley(1997)] D.L. Dunkerley. Banded vegetation: development under uniform rainfall from a simple cellular automaton model. Plant Ecol., (129):103-111, 1997.

[HilleRisLambers et al.(2001)] R. HilleRisLambers, M. Rietkerk, F. van den Bosch, H.H.T. Prins, and H. de Kroon. Vegetation pattern formation in semi-arid grazing systems. Ecology, (82):50-62, 2001.

[Hoare et al.(2004)] D.J. Hoare, I.D. Couzin, J.-G.J. Godin, and J. Krause Context-dependent group size choice in fish. Animal Behavior, 67:155164, 2004.

[Hose et al.(2000)] L.D. Hose, A.N. Palmer, M.V. Palmer, D.E. Northup, P.J. Boston, and H.R. Duchene. Microbiology and geochemistry in a hydrogen sulphide-rich karst environment. Chemical Geology, 169:399-423, 2000.

[Krause \& Tegeder(1994)] J. Krause and R.W. Tegeder. The mechanism of aggregation behavior in fish shoals: individuals minimize approach time to neighbours. Anim. Behav., (48):353-359, 1994.

[Hardenberg et al.(2001)] J. von Hardenberg, E. Meron, M. Shachak, and Y. Zarmi1, "Diversity of Vegetation Patterns and Desertification," Physical Review Letters, vol. 87, no. 19, pp. 198101-1-198101-4, Nov. 2001.

[Meron et al.(2004)] E. Meron, E. Gilad, J. von Hardenberg, M. Shachak, and Y. Zarmi. Vegetation patterns along a rainfall gradient. Chaos, Solutions and Fractals, (19):367-376, 2004.

[Pitcher et al.(1996)] T.J. Pitcher, O.A. Misund, A. Fernö, B. Totland, and V. Melle. Adaptive behaviour of herring schools in the norwegian sea as revealed by high-resolution sonar. ICES Journal of Marine Science, (53):449-452, 1996.

[Thiéry et al.(1995)] J. Thiéry, J.M. d'Herbès, and C. Valentin. A model simulating the genesis of banded vegetation patterns in niger. J. Ecol., (83):497-507, 1995. 